\title{
Crise financière, répartition des revenus et relance par les
} salaires

Financial crisis, income distribution and a wage-growth strategy

Crisis financiera, repartición de ingresos y recuperación económica a través de los salarios

\section{Marc Lavoie}

Numéro 55, automne 2013

Financiarisation du capitalisme

URI : https://id.erudit.org/iderudit/1027680ar

DOI : https://doi.org/10.7202/1027680ar

\section{Aller au sommaire du numéro}

\section{Éditeur(s)}

Athéna éditions

ISSN

0831-1048 (imprimé)

1923-5771 (numérique)

Découvrir la revue

\section{Citer cet article}

Lavoie, M. (2013). Crise financière, répartition des revenus et relance par les salaires. Cahiers de recherche sociologique, (55), 19-41.

https://doi.org/10.7202/1027680ar
Résumé de l'article

Tandis que la plupart des économistes attribuent la crise financière de 2007-2008 aux incohérences des marchés financiers, un certain nombre d'économistes, surtout hétérodoxes, associent cette crise à des éléments structurels, notamment la croissance des inégalités, mesurée par la progression de la part des profits dans le revenu national et par la dispersion des salaires. L'auteur présente les résultats d'un projet de recherche dont les différents modules ont analysé les causes de ces évolutions ainsi que les conséquences négatives de la baisse de la part des salaires pour la croissance de la demande et la croissance de la productivité. Les politiques néolibérales ont donc eu des effets néfastes pour l'économie, effets qui n'ont pu être compensés que temporairement par des artifices comme l'endettement accru des ménages et les politiques de croissance par les exportations. Il importe donc de faire marche arrière, et de revenir à des politiques favorisant le plein emploi et la relance par les salaires. 


\section{Crise financière, répartition des revenus et relance par les salaires}

MARC LAVOIE

\section{Introduction}

La crise financière qui a débuté à l'été 2007 a mené à une réévaluation des théories économiques. Alors que, avant la crise, de nombreux observateurs jugeaient que les économistes avaient enfin trouvé le moyen d'éviter les fortes fluctuations économiques et que les théories macroéconomiques dominantes étaient parvenues à un heureux consensus, la crise a montré au contraire que les opinions des économistes étaient plus que jamais éclatées. La crise a intensifié le débat sur le rôle de l'État, en particulier le débat sur le bien-fondé des politiques de relance qui ont été mises en place vers la fin de 2008 et le début de 2009 dans un grand nombre de pays. Le débat s'est prolongé quand on s'est aperçu que la crise était encore plus grave que prévu, et que nombre de gouvernements subissaient une hausse continue de leur ratio dette/PIB, en raison de la récession et de la chute de leurs recettes fiscales.

La crise a aussi relancé le débat sur l'impact de la financiarisation de l'économie, c'est-à-dire la place toujours plus importante prise par les marchés financiers laissés libres de véritables contraintes législatives et de supervision. La crise a également fait ressortir un fait qui avait été identifié auparavant par un certain nombre de chercheurs, mais sans que l'ensemble de la société y ait trop porté attention, à savoir que la part des salaires dans le 
revenu national avait considérablement chuté dans un grand nombre de pays et que la répartition des revenus était devenue plus inégalitaire. Tout cela, du moins en Amérique du Nord et dans quelques autres pays, était accompagné d'un fort endettement des ménages, ce phénomène tout comme celui de la hausse des inégalités ayant aussi été observés juste avant la Grande Dépression des années 1930, donnant ainsi un air de déjà vu à la crise présente.

On peut dire qu'il existe trois grandes explications à l'apparition de la crise financière globale, ou crise financière des subprimes, et de ses conséquences dévastatrices ${ }^{1}$. La première explication affirme que le système capitalisme fonctionne impeccablement bien, sauf lorsque les forces du marché sont entravées, et donc que si la crise est survenue c'est parce que des gouvernements ou des agences gouvernementales ont tenté de s'opposer aux lois de l'offre et de la demande. Cette explication est prônée par les économistes rattachés à l'École de Chicago, proches des enseignements de Milton Friedman, et aussi par les économistes de l'École néo-autrichienne, inspirés par les enseignements de Friedrich Hayek. On la retrouve aussi chez les macroéconomistes dits d'eau douce ${ }^{2}$, selon lesquels les marchés sont toujours à l'équilibre, ceux-ci étant souvent enseignants dans les universités situées proches des Grand Lacs.

Pour tous ces économistes, la crise financière est survenue parce que les gouvernements ont interféré avec les marchés. Par exemple, aux États-Unis, le gouvernement fédéral aurait forcé les banques américaines à consentir des prêts à des emprunteurs situés dans des communautés défavorisées, contribuant ainsi à la crise des subprimes. De plus, la Banque centrale américaine, la Réserve fédérale, aurait fixé des taux d'intérêt à court terme beaucoup trop bas à la suite de la crise boursière de 2001. Enfin, la crise financière aurait aussi été provoquée par l'obstination du gouvernement chinois à fixer son taux de change à des niveaux trop bas, ce qui aurait permis à la Banque centrale chinoise d'accumuler des montants indus de réserves de change, dont le placement sur le marché obligataire américain aurait ainsi mené à une diminution inappropriée des taux d'intérêt à long terme. Ainsi les taux d'intérêt trop bas, tant à court terme qu'à long terme, auraient incité ménages et entreprises à investir dans des projets non rentables. Pour ces économistes, les politiques de relance n'ont fait qu'empirer la situation.

1. Cette introduction s'inspire de l'introduction au volume dirigé par Marc Lavoie et Engelbert Stockhammer, Wage-Led Growth, Basingstoke, Palgrave Macmillan, 2013. Les trois grandes explications évoquées ici se retrouvent aussi dans le livre de Thomas I. Palley, From Financial Crisis to Stagnation: The Destruction of Shared Prosperity and the Role of Economics, Cambridge, Cambridge University Press, 2012. On pourrait aussi évoquer l'explication marxiste traditionnelle, selon laquelle la crise résulte de la baisse tendancielle du taux de profit.

2. Selon la distinction faite par Paul Krugman, «Economics in the crisis», 5 mars 2012, <http://krugman.blogs. nytimes.com/2012/03/05/economics-in-the-crisis/>. 
Selon la seconde explication, la crise financière est un exemple extrême de déficience de marché et d'information déficiente. Ce point de vue est surtout associé aux économistes dits Nouveaux Keynésiens, ou encore économistes d'eau salée, les plus connus de leurs représentants étant surtout localisés sur la côte est américaine, et aussi en Californie. Pour ces économistes, les marchés sont loin d'être parfaits, aussi faut-il les réglementer. Cela est particulièrement le cas des marchés financiers. Or la financiarisation de l'économie a été facilitée par la déréglementation des marchés financiers et par le manque de supervision. Cette financiarisation a été accompagnée par des changements dans le style de rémunération des chefs d'entreprise et de leurs gestionnaires, ce qui a favorisé des fraudes financières et des comportements frauduleux. De plus, certaines innovations financières ont eu des conséquences inattendues, comme la titrisation, qui permet à une institution financière de transformer des prêts, ne pouvant pas être échangés, en obligations pouvant être vendues sur les marchés financiers. La titrisation a modifié le comportement des banquiers, ceux-ci n'ayant plus grande incitation à s'assurer de la solidité de leurs emprunteurs, puisqu'ils pouvaient se débarrasser des prêts moins performants. L'existence d'une information asymétrique, ou imparfaite, juxtaposée à des innovations financières et à la dérégulation financière, aurait donc entraîné une sous-estimation systématique des risques associés aux actifs financiers et à des comportements frauduleux.

Bien qu'ils reconnaissent le bien-fondé des éléments microéconomiques avancés par les tenants de la seconde explication, certains économistes, surtout hétérodoxes, conçoivent une troisième explication de la crise financière globale. Cette troisième explication repose sur des causes structurelles, liée à l'évolution de variables macroéconomiques, tout particulièrement les inégalités de revenus ${ }^{3}$. Cette troisième explication se nourrit du constat que les politiques économiques mises de l'avant par la plupart des gouvernements ont subi des changements substantiels depuis les années 1980. Ces changements accompagnent une transformation globale de la société, qui a graduellement donné son accord à la vision néolibérale. Cette transformation s'est illustrée de diverses façons. Les gouvernements et leurs banques centrales ont progressivement abandonné l'objectif du plein emploi pour se concentrer sur celui de la stabilité des prix ; les programmes sociaux ont subi des coupures et on a restreint leur accessibilité; la finance a pris un rôle plus important, et les entreprises se sont donné pour mandat principal de satisfaire les détenteurs d'actions plutôt que de favoriser le bien-être de leur main-d'œuvre, incitant

3. Parmi les chercheurs orthodoxes, on peut citer le livre de Raghuram G. Rajan, Fault Lines: How Hidden Fractures Still Threaten the World Economy, Princeton, Princeton University Press, 2010, ainsi que le récent livre de Joseph E. Stiglitz, Le prix de l'inégalité, Paris, Éditions les liens qui libèrent, 2012. 
ainsi les entreprises à distribuer davantage de dividendes, à mettre à pied leur main-d'œuvre et à réduire leurs investissements. Tous ces changements, plus la législation qui a favorisé la mondialisation du commerce et des mouvements de capitaux, ont affaibli le pouvoir de négociation des travailleurs, du moins ceux ne faisant pas partie de la haute direction, menant ainsi à des baisses assez substantielles de la part des salaires dans le revenu national dans la grande majorité des pays. De plus, la dispersion des salaires a crû de façon substantielle, un phénomène qui peut être directement associé à la financiarisation, avec les rémunérations et les bonis extravagants qui sont versés dans le secteur financier.

La chute de la part des salaires et la croissance des inégalités de revenus ont modifié le processus de la croissance. Tandis que la croissance était autrefois tirée par les salaires, lesquels progressaient approximativement au même rythme que la productivité par travailleur, la croissance au cours des deux dernières décennies a reposé sur deux piliers, d'une part la croissance de l'endettement des ménages (la croissance menée par l'endettement) et d'autre part les restrictions salariales, permettant une meilleure compétitivité et la possibilité d'exporter (la croissance menée par les exportations). Ces régimes d'accumulation sont cependant insoutenables, car par définition tous les pays ne peuvent avoir un solde commercial en surplus, tandis que l'endettement des ménages ne peut augmenter indéfiniment, surtout si leurs revenus stagnent. Il est donc clair que les gouvernements doivent reconsidérer ces deux types de régime d'accumulation, et mettre en place des conditions permettant de revenir au régime d'accumulation tiré par les salaires. Les gouvernements doivent remettre en question les vérités inattaquables ou la sagesse conventionnelle comme l'aurait dit John Kenneth Galbraith, plus particulièrement la croyance selon laquelle la modération salariale et des marchés du travail dénués d'entraves mèneront à une économie plus performante et plus productive.

Certaines organisations internationales reconnaissent maintenant les dangers inhérents à une trop grande inégalité des revenus. C'est le cas notamment de la Conférence des Nations Unies sur le Commerce et le Développement $(\mathrm{CNUCED})^{4}$ et du Bureau International du Travail (BIT), lequel a commandé une étude en six modules permettant de comprendre les causes et les conséquences de ces changements dans la répartition des revenus, et d'en tirer certaines leçons de politique économique.

4. Voir notamment ses Rapport sur le commerce et le développement de 2010, 2011 et 2012. <http://unctad.org/ $\mathrm{fr} /$ pages/Publications/TradeandDevelopmentReport.aspx>. 
Le but du présent article est de présenter les principaux résultats de cette recherche poursuivie avec des collègues européens au cours des deux dernières années. Nous commençons par rappeler quelques données qui vont motiver notre étude, puis nous présenterons le cadre conceptuel qui a nourri ces études. Enfin, nous nous pencherons sur les études empiriques permettant de saisir l'impact de la baisse de la part des salaires sur l'activité économique.

\section{Quelques grandes tendances}

Nous avons affirmé en introduction que la part des revenus salariaux avait baissé au cours des vingt dernières années, et nous avons affirmé que la répartition des revenus était devenue plus inégale durant cette période. Les tableaux 1 et 2 illustrent la généralité de ces deux phénomènes, principalement pour les pays faisant partie du G20, lesquels représentent près de $80 \%$ de la production mondiale. La dernière colonne du tableau 1 montre que la part des salaires a diminué dans tous les pays au cours de la dernière décennie, et si on examine les données pour les années 1980, on constate que c'est encore plus le cas sur les deux dernières décennies pour les pays pour lesquels on dispose de données. Quant aux inégalités de revenus, le tableau 2 en donne une illustration, en se penchant sur la part des revenus obtenus par les individus situés dans le premier centile, le fameux $1 \%$ de la population dont on parle tant depuis l'avènement du mouvement Occupons le Canada, inspiré du mouvement des indignés de Wall Street ${ }^{5}$. On observe que cette part a crû dans tous les pays, sauf aux Pays-Bas, et que cette part a crû de façon considérable dans les pays anglo-saxons et en Argentine.

En économie hétérodoxe (économie marxiste, économie post-keynésienne), l'une des croyances les plus établies, c'est que la propension à consommer d'une économie est d'autant plus faible que les revenus sont distribués aux gens les plus riches et que la répartition fonctionnelle favorise les profits. Une telle répartition des revenus conduit donc à une réduction de la consommation et à un affaiblissement de la demande globale. Pourtant, jusqu'à la crise financière globale de 2008, on ne peut pas dire qu'il y a eu un effondrement de l'activité économique. Comment peut-on expliquer cette

5. Des données plus complètes pour le Canada sont fournies par Michael Veall, «A new gilded age Recent trends in top income shares in Canada», 6 février 2012, <www.socialsciences.uottawa.ca/irpe-epri/eng/documents/ VeallSlidesFeb2012.pdf>. Voir aussi l'article récent de N. Fortin, D. A. Green, T. Lemieux, K. Milligan et W. Craig Riddell, «Canadian inequality: recent developments and policy options», Canadian Public Policy, vol. 38, n², 2012, p. 121-145, ainsi que le rapport de l'Institut Broadbent, «Towards a more equal Canada: A report on Canada's economic and social inequality», octobre 2012, <www.broadbentinstitute.ca/sites/default/files/documents/ towards_a_more_equal_canada.pdf>. 


\section{Tableau 1}

Part des salaires dans le revenu national, en pourcentage, pays du G20, valeur moyenne au cours du cycle, début 1980 à 2008

\begin{tabular}{|l|c|c|c|c|}
\hline \multicolumn{1}{|c|}{ Pays } & $\begin{array}{c}\text { A. Début } \\
\mathbf{1 9 8 0} \\
\text { - fin 1990 }\end{array}$ & $\begin{array}{c}\text { B. Début } \\
\mathbf{1 9 9 0} \\
\text { - début } \mathbf{2 0 0 0}\end{array}$ & $\begin{array}{c}\text { C. Début } \\
\mathbf{2 0 0 0} \\
\mathbf{- 2 0 0 8}\end{array}$ & $\begin{array}{c}\text { Écart (C - B), } \\
\text { en points de } \\
\text { pourcentage }\end{array}$ \\
\hline Afrique du Sud & 56,65 & 54,87 & $50,18^{c}$ & $-4,69$ \\
\hline Allemagne & 67,11 & 66,04 & 63,37 & $-2,67$ \\
\hline Arabie saoudite & $\ldots$ & $\ldots$ & $\ldots$ & $\ldots$ \\
\hline Argentine & $\ldots$ & 38,42 & $32,79^{c}$ & $-5,63$ \\
\hline Australie & 66,70 & 65,76 & 62,57 & $-3,19$ \\
\hline Brésil & $\ldots$ & 43,33 & $39,64 c)$ & $-3,69$ \\
\hline Canada & 66,89 & 67,79 & 63,75 & $-4,05$ \\
\hline Chine & 15,58 & 13,11 & 10,82 & $-2,28$ \\
\hline Corée du Sud & 81,62 & 80,53 & 76,97 & $-3,56$ \\
\hline États-Unis & 68,20 & 67,12 & 65,87 & $-1,25$ \\
\hline France & 71,44 & 66,88 & 65,87 & $-1,01$ \\
\hline Inde & 34,03 & 32,25 & $32,18^{c}$ & $-0,07$ \\
\hline Indonésie & $\ldots$ & $\ldots$ & $\ldots$ & $\ldots$ \\
\hline Italie & 68,70 & 63,25 & 62,37 & $-0,88$ \\
\hline Japon & 72,38 & 70,47 & 65,75 & $-4,73$ \\
\hline Mexique & $\ldots$ & 46,35 & 46,16 & $-0,19$ \\
\hline Royaume-Uni & 72,98 & 71,99 & 70,73 & $-1,26$ \\
\hline Russie & $\ldots$ & 45,87 & $45,56^{c}$ & $-0,31$ \\
\hline Turquie & 48,07 & 54,12 & 50,34 & $-3,78$ \\
\hline
\end{tabular}

Source: Eckhard Hein et Matthias Mundt, "Financialisation and the requirements and potentials for wage-led recovery - a review focussing on the G20», Conditions of Work and Employment Series working paper No 37, Bureau International du Travail, Genève, 2012.

contradiction? Selon Eckhard Hein et Matthias Mundt ${ }^{6}$, divers pays ont réagi différemment à cette évolution. Dans certains pays, notamment les pays anglo-saxons, c'est un régime d'accumulation par la dette, plus précisément la dette des ménages, qui a permis de compenser la hausse des inégalités de revenus. Dans ces pays, la demande globale et la consommation ont été

6. Eckhard Hein et Matthias Mundt, «Financialisation and the requirements and potentials for wage-led recovery - a review focussing on the G20», Conditions of Work and Employment Series working paper No 37, Bureau International du Travail, Genève, 2012. 
Tableau 2

La part du revenu national du $1 \%$ des revenus supérieurs, milieu des années 1970 au milieu des années 2000

\begin{tabular}{|c|c|c|c|}
\hline Pays & $\begin{array}{c}\text { Milieu des } \\
\text { années } 1970\end{array}$ & $\begin{array}{c}\text { Milieu des } \\
\text { années } 2000\end{array}$ & $\begin{array}{l}\text { Changement, } \\
\text { en points de pourcentage }\end{array}$ \\
\hline \multicolumn{4}{|l|}{ Pays du G20 } \\
\hline Allemagne ${ }^{b)}$ & 10,4 & 12,1 & $+1,7$ \\
\hline Argentine ${ }^{b)}$ & 9,9 & 16,8 & $+6,9$ \\
\hline Australie a) $^{\text {a) }}$ & 5,0 & 9,7 & $+4,7$ \\
\hline (anada ${ }^{\text {a) }}$ & 8,2 & 12,8 & $+4,6$ \\
\hline Chine $^{\text {b) c) }}$ & 2,6 & 5,9 & $+3,3$ \\
\hline États-Unis a) & 7,9 & 18,0 & $+10,1$ \\
\hline France a) & 8,2 & 8,7 & $+0,5$ \\
\hline Inde b) d) & 7,0 & 9,5 & $+2,5$ \\
\hline Indonésie ${ }^{\text {b) e) }}$ & 7,2 & 9,1 & $+1,9$ \\
\hline Italie a) & 7,0 & 9,2 & $+2,2$ \\
\hline Japon ${ }^{\text {a) }}$ & 6,9 & 9,0 & $+2,1$ \\
\hline Royaume-Uni ${ }^{\text {b) }}$ & 6,1 & 14,3 & $+8,2$ \\
\hline \multicolumn{4}{|l|}{ Autres pays ${ }^{\text {a) }}$} \\
\hline Danemark & 4,0 & 4,3 & $+0,3$ \\
\hline Finlande & 5,7 & 8,1 & $+2,4$ \\
\hline Irlande & 5,8 & 9,8 & $+4,0$ \\
\hline Pays-Bas & 6,1 & 5,4 & $-0,7$ \\
\hline Nouvelle-Zélande & 6,7 & 9,5 & $+2,8$ \\
\hline Norvège & 5,4 & 8,2 & $+2,8$ \\
\hline Portugal & 7,1 & 9,5 & $+2,4$ \\
\hline Espagne & 7,6 & 8,8 & $+1,2$ \\
\hline Suède & 5,0 & 6,9 & $+1,9$ \\
\hline
\end{tabular}

Notes: c) la première donnée provient du milieu des années 1980; d) la seconde donnée provient de la fin des années 1990; e) la première donnée provient du début des années 1980.

Source: a) Perspectives de l'emploi de l'OCDE 2012, document complémentaire au chapitre 3, tableau 3.A2.1, <www.oecd.org/els/employmentpoliciesanddata/Chapter\%203\%20web\%20annex.pdf >; b) The world top incomes database, <http://g-mond.parisschoolofeconomics.eu/topincomes/\#Database>. 
soutenues par l'endettement accru des ménages et par la transformation du mode de fonctionnement des entreprises due au phénomène de la financiarisation. Dans ces pays, les entreprises ont réduit leurs investissements en capital fixe, en partie à cause des modifications à la gouvernance des entreprises, davantage axées sur la valeur actionnariale à court terme et sur des taux de rendement cibles élevés, à cause notamment des bonus à la performance versée aux chefs d'entreprise. Cette baisse des dépenses d'investissement a cependant pour contrepartie la hausse des dividendes versés aux actionnaires ainsi que la hausse de la valeur des actions suscitant des gains en capital. Ces deux hausses permettent de soutenir les dépenses de consommation, ellesmêmes renforcées par l'accès facile au crédit qui est une caractéristique des économies anglo-saxonnes, notamment l'accès au crédit immobilier.

Ainsi, les gains en capital sur le marché boursier ont été renforcés par les gains en capital sur les marchés immobiliers, qui ont à leur tour suscité des dépenses de consommation. Tout cela a créé, temporairement du moins, un cercle vertueux, toutes ces dépenses de consommation suscitées par les dividendes élevés et l'accroissement des taux d'endettement des ménages justifiant les taux de rendement cibles élevés, notamment le fameux taux de rendement de $15 \%$ sur le capital propre (return on equity), malgré la faiblesse de l'investissement réel. Cependant, comme l'illustre le tableau 3, les pays ayant connu de forts taux d'augmentation de l'endettement de leurs ménages se trouvent aussi fréquemment être les pays subissant un déficit de leur compte courant, ces pays important davantage qu'ils n'exportent.

Mais tandis que les ménages américains réagissaient à la baisse de leurs revenus salariaux en augmentant leur endettement et en diminuant leurs taux d'épargne de façon permanente, tentant ainsi de conserver leur place dans la hiérarchie du consommateur américain tout en tentant d'imiter le comportement de leurs voisins plus fortunés, les ménages dans d'autres pays réagissaient tout autrement. Till van Treeck et Simon Mundt ${ }^{7}$ montrent dans leur étude que la consommation des ménages allemands et chinois s'est relativement détériorée, en partie à cause de l'effet déjà identifié plus haut, à savoir qu'une répartition des revenus plus inégalitaire et plus favorable aux profits réduit la consommation et augmente l'épargne, mais aussi parce que les taux d'épargne des ménages allemands et chinois étaient en hausse. Dans ces pays, plus particulièrement en Chine, l'accès au crédit est moins facile, si bien que la détérioration du filet de protection sociale, incluant la santé, a incité les ménages à épargner davantage. En Allemagne, les revenus réels

7. Simon Sturn et Till van Treeck, «Income inequality as a cause of the Great Recession? A survey of current debates», Conditions of Work and Employment Series working paper No 39, Bureau International du Travail, Genève, 2012. 
stagnants ainsi que les réformes des avantages sociaux des travailleurs et la déréglementation du marché du travail qui protège maintenant moins bien le travailleur ont créé de l'inquiétude chez les ménages, si bien que cette insécurité, comme en Chine, a mené à une augmentation des taux d'épargne. Ainsi cette baisse de la demande interne, à laquelle s'est ajouté l'accroissement de la compétitivité externe de l'Allemagne, grâce au gel des salaires nominaux allemands, a induit une hausse substantielle du surplus du solde commercial et du solde du compte courant en Allemagne. Cela est aussi illustré au haut du tableau 3 qui montre que, en règle générale (mais pas toujours), les pays où l'endettement des ménages s'est peu accru sont aussi les pays qui bénéficient d'un solde du compte courant positif et substantiel.

On peut se demander pourquoi la répartition des revenus a évolué dans un sens si favorable aux plus riches et aux revenus du capital, un phénomène qu'on a observé non seulement dans les pays industrialisés mais aussi dans les pays en émergence. Selon l'économie dominante, la répartition des revenus est avant tout une affaire de technologie, la théorie néoclassique affirmant que dans un marché concurrentiel chaque agent est rémunéré selon la valeur de sa productivité marginale. L'explication traditionnelle de cette évolution est que le progrès technique a été favorable au capital, ce qui a donc accru sa rémunération, et que les nouvelles technologies requièrent davantage de compétences, ce qui favorise les détenteurs de diplômes. Le point de vue alternatif, qui est celui défendu par Engelbert Stockhammer et la grande majorité des économistes hétérodoxes, notamment les économistes institutionnalistes que l'on retrouve surtout maintenant dans les écoles de relations industrielles, c'est que la répartition du revenu est avant tout affaire de pouvoir de négociation. Ainsi, selon ceux-ci, la mondialisation, la financiarisation, l'abandon des politiques de plein emploi et l'affaiblissement de l'État providence ont de façon générale conduit à une diminution du pouvoir de négociation des travailleurs et conséquemment à une réduction de la part des salaires et à une réduction de la part des salaires versés aux employés qui ne sont pas des gestionnaires ou des superviseurs.

Stockhammer ${ }^{8}$ se livre à une analyse économétrique qui a pour objectif d'évaluer l'impact sur la répartition fonctionnelle des changements liés à la technologie et des changements liés au pouvoir de négociation. Pour les pays avancés, il constate que des taux de croissance rapides augmentent la part des profits, et que des taux de chômage élevés réduisent la part des salaires, les travailleurs ayant alors moins de leviers pour négocier. Stockham-

8. Engelbert Stockhammer, «Why have wages fallen? A panel analysis of the determinants of functional income distribution », Conditions of Work and Employment Series working paper No 35, Bureau International du Travail, Genève, 2012. 


\section{Tableau 3}

\section{Déséquilibres internationaux et accroissements des taux d'endettement des ménages}

\begin{tabular}{|c|c|c|c|}
\hline & Pays & $\begin{array}{l}\text { Solde du compte } \\
\text { courant (a) }\end{array}$ & $\begin{array}{c}\text { Accroissement des taux } \\
\text { d'endettement } \\
\text { des ménages (b) }\end{array}$ \\
\hline \multirow{8}{*}{$\begin{array}{l}\text { Pays avec de forts } \\
\text { surplus du compte } \\
\text { courant }\end{array}$} & Suisse & $+11,8$ & +5 \\
\hline & Russie & $+7,5$ & +9 \\
\hline & Pays-Bas & $+6,7$ & +33 \\
\hline & Chine & $+6,3$ & +8 \\
\hline & Allemagne & $+5,4$ & -11 \\
\hline & Japon & $+3,6$ & -7 \\
\hline & Autriche & $+2,9$ & +8 \\
\hline & Corée du Sud & $+2,5$ & +32 \\
\hline \multirow{8}{*}{$\begin{array}{l}\text { Pays avec de forts } \\
\text { déficits du compte } \\
\text { courant }\end{array}$} & Grèce & $-10,2$ & +35 \\
\hline & Portugal & $-9,9$ & +27 \\
\hline & Espagne & $-6,8$ & +34 \\
\hline & États-Unis & $-4,7$ & +26 \\
\hline & Irlande & $-2,6$ & +63 \\
\hline & Royaume-Uni & $-2,3$ & +32 \\
\hline & Italie & $-2,1$ & +18 \\
\hline & Australie & $-2,0$ & +42 \\
\hline
\end{tabular}

Notes: a) Solde du compte courant, en pourcentage du PIB, moyennes 2003-2010. b) Accroissement de l'endettement des ménages, en points de pourcentage du PIB, entre 2000 et 2008. L'Irlande débute en 2001; la Suisse débute en 1999 et termine en 2007.

Source: Marc Lavoie et Engelbert Stockhammer, "Wage-led growth: Concept, theories and policies", Conditions of Work and Employment Series working paper No 41, Bureau International du Travail, Genève, 2012.

mer constate aussi que le taux de syndicalisation et l'importance relative des dépenses du gouvernement ont un impact positif sur la part des salaires. Par contre, comme on pourrait s'y attendre, une hausse de la mondialisation et de la financiarisation, mesurée par la somme des ratios par rapport au PIB des importations et des exportations et par la somme de l'actif et du passif financier d'un pays par rapport à son PIB, a un effet défavorable sur la part des salaires.

Lorsqu'on tient compte de tous ces effets, les variables liées au changement technologique, mesurées par le taux de capitalisation (le rapport entre 
capital et travail) et la part du secteur des nouvelles technologies dans l'économie, n'ont plus qu'un impact négatif très mineur sur la part des salaires. Lorsque Stockhammer refait le même exercice pour un ensemble de 71 pays avancés et en développement, en utilisant un ensemble de variables restreint, il retrouve essentiellement les mêmes résultats, la mondialisation et les coupures dans l'État providence ayant le plus fort impact négatif sur la part des profits. Quant au progrès technologique, mesuré ici par l'évolution de la part du secteur manufacturier par rapport au secteur agricole, il n'a plus aucun effet sur la part des salaires, si ce n'est un effet positif! Ainsi, contrairement aux chercheurs traditionnels qui prétendent que l'évolution de la part des salaires et des inégalités est un phénomène inéluctable associé au progrès technique, Stockhammer conclut plutôt que la baisse des salaires au cours des deux dernières décennies s'explique surtout par un désengagement de l'État par rapport à l'objectif du plein emploi et par la réduction du pouvoir de négociation des travailleurs, causé notamment par des phénomènes soutenus par les États, à savoir la mondialisation et la financiarisation. Les changements observés dans la répartition du revenu ne sont donc ni inéluctables ni irréversibles.

On peut cependant se demander pourquoi il serait nécessaire de renverser la tendance, et pourquoi des hausses salariales seraient une bonne affaire pour l'économie. Quand on regarde les choses d'un point de vue microéconomique, au niveau de l'entreprise individuelle, il est évident qu'une hausse des salaires est un coût pour l'entreprise et un obstacle à la rentabilité et à la pérennité de l'entreprise. Cependant, il est important d'aller au-delà de la vision microéconomique et de considérer les salaires autrement que comme un coût. Il faut examiner les salaires sous l'angle macroéconomique. Les salaires sont également des revenus, qui constituent une composante importante de la demande globale à travers leur consommation. De plus, comme nous allons le voir, les hausses de salaires peuvent avoir des effets favorables du côté de l'offre, en générant des hausses de productivité. Ce sont ces possibilités que nous allons maintenant examiner et qui justifient une relance de l'économie mondiale par les salaires. En effet, dans une section subséquente, nous allons montrer que la plupart des économies du G20 participent d'un régime de croissance mené par les salaires.

\section{Croissance et répartition : une conceptualisation}

Avant d'examiner pourquoi la hausse rapide des salaires pourrait avoir un impact favorable sur l'économie, il faut tout d'abord faire quelques distinc- 
tions et procéder à quelques définitions9. Nous allons définir comme politiques favorables au capital les politiques qui mènent à une baisse tendancielle de la part des salaires dans le revenu national. Ces politiques comprennent les restrictions et les obstacles à la syndicalisation, l'affaiblissement du processus de négociation collective, les restrictions aux grèves, l'affaiblissement des protections sociales, la diminution de la valeur réelle du salaire minimum ou de sa valeur par rapport au salaire médian. Les promoteurs des politiques favorables au capital font habituellement valoir que ces politiques vont améliorer la flexibilité et l'efficacité de l'économie; ils font rarement valoir que ces politiques vont accroître les revenus du capital. Il en va de même des politiques fiscales qui modifient la répartition secondaire du revenu, comme la diminution du taux d'imposition des sociétés ou du taux d'imposition sur les gains en capital. Les politiques favorables aux travailleurs vont évidemment dans le sens inverse, comme le montre le tableau 4.

Il nous faut maintenant considérer une autre question. Sachant par exemple que les politiques de répartition sont favorables au capital et mènent à un accroissement de la part des profits dans le revenu national, quel est l'impact économique de ce changement? Quel est l'impact sur la demande globale à court terme, ou sur le taux de croissance de la demande globale, ou encore quel est l'impact sur le taux de croissance de la productivité? Si l'augmentation de la part des profits a des répercussions favorables sur ces diverses variables de l'économie, on dira que l'économie se situe dans un régime économique mené par les profits. Dans le cas contraire, si l'augmentation de la part des profits a un impact négatif sur l'économie, on dira que cette économie est dans un régime économique mené par les salaires. Évidemment, on peut aussi considérer ce qui arrive quand c'est la part des salaires qui est en hausse, comme l'illustre le tableau 5.

Ce qu'il est important de comprendre, c'est que le régime économique dans lequel se trouve une économie est déterminé par des facteurs structurels et par le comportement des divers agents de l'économie, sur lesquels les politiques gouvernementales ont peu de prise, sinon à très long terme. Nous reviendrons plus loin sur les déterminants de ces régimes économiques. Par contre, le gouvernement peut influencer l'évolution de la répartition des revenus, par ses politiques et sa législation, ainsi que nous l'avons rappelé dans la section précédente.

Qu'arrive-t-il maintenant si l'on croise les tableaux 4 et 5 , pour obtenir le tableau 6? Seuls deux croisements engendrent une croissance stable et

9. Cette section et ses tableaux s'inspirent du texte de Marc Lavoie et Engelbert Stockhammer, «Wage-led growth: Concept, theories and policies», Conditions of Work and Employment Series working paper No 41, Bureau International du Travail, Genève, 2012. 
Tableau 4

Politiques favorable aux salariés et favorable au capital

\begin{tabular}{|c|c|c|c|}
\hline & \multicolumn{2}{|c|}{ Politiques de répartition } & \multirow{2}{*}{ Autres facteurs } \\
\hline & Pro-capital & Pro-travailleurs & \\
\hline Politiques & $\begin{array}{l}\text { «Flexibilité des marchés } \\
\text { du travail» } \\
\text { Abolir le salaire } \\
\text { minimum } \\
\text { Affaiblir la négociation } \\
\text { collective } \\
\text { Imposer les restrictions } \\
\text { salariales }\end{array}$ & $\begin{array}{l}\text { «L'État providence» } \\
\text { Augmenter le salaire } \\
\text { minimum relatif } \\
\text { Renforcer la négociation } \\
\text { collective }\end{array}$ & $\begin{array}{l}\text { Changements } \\
\text { technologiques } \\
\text { Mondialisation } \\
\text { Financiarisation }\end{array}$ \\
\hline Résultats & $\begin{array}{l}\text { Croissance faible } \\
\text { des salaires } \\
\text { Part des salaires } \downarrow \\
\text { Dispersion accrue } \\
\text { des salaries }\end{array}$ & $\begin{array}{l}\text { Croissance des salaires } \\
\text { réels } \\
\text { Part des salaires stable } \\
\text { (ou } \uparrow \text { ) } \\
\text { Dispersion réduite } \\
\text { des salaires }\end{array}$ & \\
\hline
\end{tabular}

Tableau 5

Définition des régimes économiques menés par les profits et par les salaires

\begin{tabular}{|c|c|c|c|}
\hline & & \multicolumn{2}{|c|}{ Impact sur l'économie } \\
\hline & & Expansion & Contraction \\
\hline \multirow{2}{*}{$\begin{array}{l}\text { Changement dans } \\
\text { la répartition des } \\
\text { revenus imposé } \\
\text { à la société }\end{array}$} & $\begin{array}{l}\text { Une hausse } \\
\text { de la part des } \\
\text { profits }\end{array}$ & $\begin{array}{l}\text { Régime } \\
\text { économique mené } \\
\text { par les profits }\end{array}$ & $\begin{array}{l}\text { Régime } \\
\text { économique mené } \\
\text { par les salaires }\end{array}$ \\
\hline & $\begin{array}{l}\text { Une baisse } \\
\text { de la part des } \\
\text { profits }\end{array}$ & $\begin{array}{l}\text { Régime } \\
\text { économique mené } \\
\text { par les salaires }\end{array}$ & $\begin{array}{l}\text { Régime } \\
\text { économique mené } \\
\text { par les profits }\end{array}$ \\
\hline
\end{tabular}


Tableau 6

Viabilité des régimes de croissance

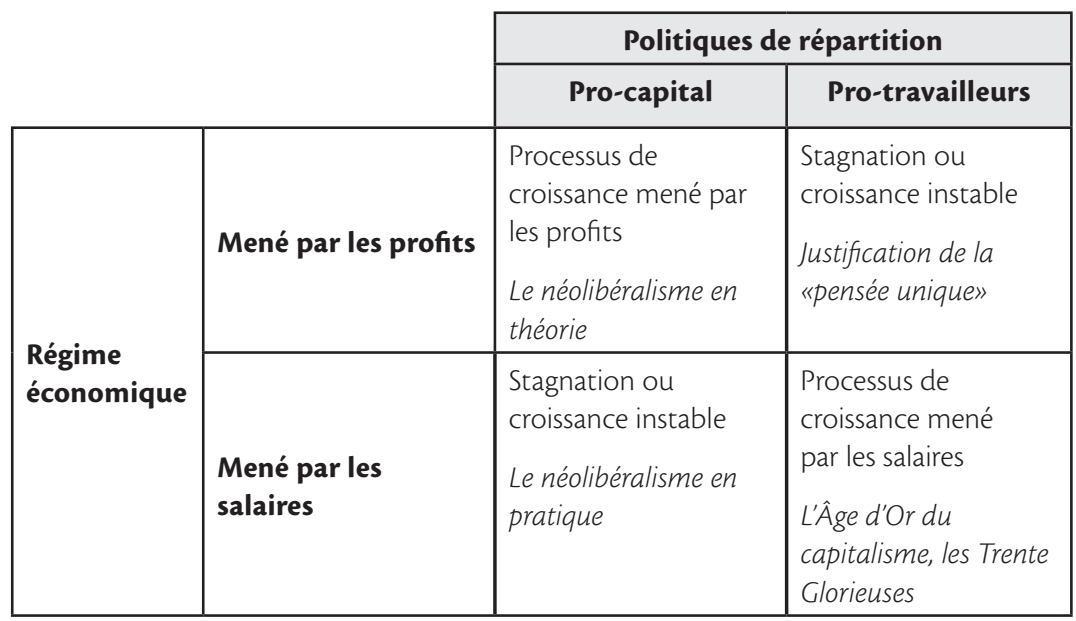

soutenue: dans un régime économique tiré par les profits, il est préférable d'avoir des politiques de répartition favorisant le capital; par contre dans un régime économique tiré par les salaires, il faut des politiques de répartition favorisant les travailleurs. Le premier croisement correspond aux promesses du néolibéralisme: en donnant plus aux riches et aux détenteurs du capital, tous les travailleurs finiront par en bénéficier. Le second croisement correspond aux trente années qui ont suivi la Seconde Guerre mondiale. Dans les deux autres cas, les contradictions entre les politiques poursuivies par l'État et le régime économique inhérent au pays entraîneront une croissance au ralenti ou des déséquilibres néfastes à la croissance. Les auteurs des six modules commandés par le BIT croient justement que les vingt dernières années ont été caractérisées par des politiques de répartition pro-capital, faites dans des pays régis le plus souvent par un régime économique mené par les salaires. Les politiques néolibérales ont donc, en pratique, donné lieu soit à une croissance au ralenti soit à l'apparition de déséquilibres systémiques, car une croissance économique rapide nécessitait des éléments amplificateurs : la croissance de l'endettement des ménages ou alors des soldes commerciaux en surplus, comme nous l'avons expliqué dans la section précédente.

La crise financière actuelle risque de nous plonger encore davantage dans un processus de croissance inopérant. En effet, la crise affaiblit le pouvoir de négociation des travailleurs et elle a entraîné l'apparition de substantiels déficits budgétaires, même dans les pays ou les régions qui pouvaient se 
targuer de finances publiques saines. Les pressions à la baisse sur les salaires, qui vont se matérialiser par des gels de salaire malgré la hausse continue des prix, vont donc opérer autant dans le secteur privé que dans le secteur public. Par ailleurs, malgré l'indignation des populations et les vagues promesses de réglementation du secteur financier, il ne semble pas encore que le processus de financiarisation de l'économie soit près de s'arrêter. Il est donc impératif de reconsidérer les politiques néolibérales favorables au capital.

\section{Les régimes de demande}

Nous avons affirmé que les changements dans la répartition des revenus pouvaient avoir des effets favorables ou défavorables sur l'économie. Traditionnellement, les économistes, depuis les travaux poursuivis par les économistes de l'École de la régulation française, notamment les travaux de Robert Boyer ${ }^{10}$, distinguent les effets sur la demande et les effets sur la productivité. Nous verrons que ces effets finissent par s'enchevêtrer, mais commençons par les effets sur la demande globale. Quels sont les déterminants des régimes de demande?

Le produit intérieur brut d'un pays, son PIB, est la somme de quatre composantes: les dépenses de consommation, les dépenses d'investissement, les exportations nettes (exportations moins importations) et les dépenses du gouvernement (les transferts étant exclus). L'impact d'un changement dans la répartition du revenu dépendra donc de la somme de son impact sur ces quatre composantes. En pratique, les recherches portent sur l'impact des trois premières composantes. Le tableau 7 résume les déterminants structurels qui vont faire qu'une économie est située dans un régime de demande menée par les salaires ou par les profits ${ }^{11}$. En général, une répartition plus favorable aux salaires va permettre une augmentation de la consommation, car en général, une plus grande partie des salaires que des profits est consommée (le reste étant épargné). De façon technique, on dit que la propension à consommer sur les salaires est plus élevée que la propension à consommer sur les profits. L'écart entre les deux propensions à consommer semble se situer autour de 0,40 ou 0,45 selon les estimations statistiques les plus récentes ${ }^{12}$. Cet écart est donc substantiel, et laisse à penser que les économies sont souvent en régime de demande mené par les salaires. Cet écart est dû au fait

10. Robert Boyer, «Formalizing growth regimes within a regulation approach», dans G. Dosi, C. Freeman, R. Nelson, G. Silverberg et L. Soete (dir.), Technical Change and Economic Theory, Londres et New York, Pinter Publishers, 1988, p. 608-630.

11. Ce tableau s'inspire du modèle néo-kaleckien de croissance. On en trouvera une présentation simple dans le livre de Marc Lavoie, L'Économie postkeynésienne, Paris, La Découverte, 2004; et une présentation plus complète par Oliver Allain, «La modération salariale: le point de vue (néo)-kaleckien», Revue économique, vol. 60, n 1, janvier 2009, p. 81-108.

12. Voir Servaas Storm et C.W.M. Naastepad, Macroeconomics Beyond the NAIRU, Cambridge, Harvard University Press, 2012, p. 129. 


\section{Tableau 7}

La structure économique des régimes de demande menés par les profits et par les salaires

\begin{tabular}{|l|l|l|}
\hline \multirow{1}{*}{$\begin{array}{c}\text { Structure } \\
\text { économique }\end{array}$} & \multicolumn{2}{c|}{ Régime de demande } \\
\hline \multirow{5}{*}{ Consommation } & \multicolumn{1}{|c|}{ Mené par les profits } & \multicolumn{1}{c|}{ Mené par les salaires } \\
\cline { 2 - 3 } & $\begin{array}{l}\text { Faibles différences entre les } \\
\text { propensions à consommer }\end{array}$ & $\begin{array}{l}\text { La propension à consommer sur } \\
\text { les salaires est bien plus élevée } \\
\text { que la propension à consommer } \\
\text { sur les profits }\end{array}$ \\
\cline { 2 - 3 } Investissement & $\begin{array}{l}\text { L'investissement réagit } \\
\text { fortement à la profitabilité } \\
\text { mais réagit peu aux ventes }\end{array}$ & $\begin{array}{l}\text { L'investissement réagit peu } \\
\text { à la profitabilité, mais réagit } \\
\text { fortement aux ventes }\end{array}$ \\
\cline { 2 - 3 } Exportations nettes & $\begin{array}{l}\text { Économie très ouverte, } \\
\text { avec des exportations } \\
\text { sensibles aux variations de } \\
\text { prix et des importations } \\
\text { variant fortement avec la } \\
\text { production intérieure }\end{array}$ & $\begin{array}{l}\text { Économie relativement fermée, } \\
\text { avec des exportations réagissant } \\
\text { peu aux variations de prix et des } \\
\text { importations variant peu avec } \\
\text { la production intérieure }\end{array}$ \\
\hline
\end{tabular}

qu'une partie des profits engrangés par les entreprises est automatiquement épargnée par les entreprises (ce sont les profits non distribués), tandis que les profits distribués aux ménages sous la forme de paiements en intérêts et en dividendes sont souvent accaparés par des gens riches, qui consomment une fraction réduite de leurs revenus par comparaison aux individus faisant partie des déciles de la classe moyenne ou des déciles inférieurs.

Par contre, une répartition plus favorable aux travailleurs va réduire la profitabilité des entreprises, pour un taux d'utilisation des capacités productives donné. Cela risque donc de faire diminuer les dépenses d'investissement des entreprises, d'autant plus que les entreprises réagissent aux indices de rentabilité. Par contre, si leurs investissements dépendent fortement de leurs ventes et de leur taux d'utilisation des capacités, il est possible que cet effet négatif soit annulé, car la hausse de la part des salaires va entraîner une hausse des ventes aux consommateurs, ce qui pourrait inciter les entreprises à augmenter leurs capacités productives. Enfin, en général, on peut dire que la hausse de la part des salaires a aussi un effet négatif sur la troisième composante du PIB, les exportations nettes, car la hausse des salaires va souvent mener à une hausse des prix, ce qui risque d'affecter négativement les exportations. 
Il est essentiel de bien distinguer les régimes de demande selon que l'on ne considère d'une part que la demande intérieure, et d'autre part selon que l'on considère la demande totale, tant celle provenant de l'intérieur que celle provenant de l'étranger. Cette distinction est évidemment fondamentale, car elle nous évite de commettre une erreur de composition. En effet, nous allons voir qu'un certain nombre de pays se situent dans un régime de demande totale mené par les profits bien que leur régime de demande intérieure soit mené par les salaires. Donc, pour ce type de pays, il est préférable de poursuivre des politiques de répartition favorables aux profits. Cependant, nous savons aussi que tous les pays ne peuvent pas bénéficier d'exportations nettes positives, puisque la somme de soldes commerciaux de tous les pays doit nécessairement être égale à zéro. À un ensemble de pays avec solde commercial en surplus doit correspondre un autre ensemble de pays avec solde commercial en déficit. Les politiques de répartition pro-capital poursuivies par un pays auront donc des conséquences négatives pour les partenaires de ce pays. De fait, si tous les pays se situent dans un régime de demande intérieure mené par les salaires avec un régime de demande totale mené par les profits, l'ensemble de l'économie mondiale fonctionnera sous la forme d'un régime de demande mené par les salaires. Si ces pays poursuivent tous, en toute logique individuelle, des politiques de répartition favorables aux profits, l'économie mondiale tombera en récession, puisque la demande globale de l'économie mondiale est alors tirée par les salaires!

Les toutes dernières recherches empiriques, faites par Özlem Onaran et Giorgos Galanis ${ }^{13}$ dans le cadre de l'un des modules, confirment ce jugement. Le tableau 8 résume leurs principaux résultats. On y constate effectivement que tous les pays du G20, qui représentent $80 \%$ de la production mondiale, sont en régime de demande intérieure tirée par les salaires. Autrement dit, quand on y augmente la part des profits, cela mène à une baisse de la demande intérieure (la colonne d), l'impact négatif sur la consommation surpassant l'impact positif (parfois nul) sur l'investissement. On constate aussi, quand on tient compte de l'effet d'une augmentation de la part des profits sur le commerce extérieur, qu'un grand nombre de ces pays, même l'Allemagne, sont en régime de demande totale tirée par les salaires (la colonne e), le Canada, l'Australie et des pays semi-industrialisés faisant partie des exceptions. Cependant, lorsque tous les pays du G20 augmentent la part des profits de 1 point de pourcentage, le taux de croissance du PIB de l'ensemble des pays du G20 diminue de 0,36 point de pourcentage. Ainsi, il est clair que toute politique généralisée de modération salariale provoquée par la crise

13. Özlem Onaran et Giorgos Galanis, «Is aggregate demand wage-led or profit-led? National and global effects», Conditions of Work and Employment Series working paper No 40, Bureau International du Travail, Genève, 2012. 
financière et la crise budgétaire en Europe aura des conséquences funestes sur l'économie mondiale.

Onaran et Galanis montrent que tous les pays, sauf la Chine et l'Afrique du Sud, vont pâtir des conséquences d'une augmentation généralisée de la part des profits, même si certains de ces pays, comme le Canada, pourraient tirer avantage d'une augmentation de la part des profits qui serait limitée à leur propre pays. Dans le cas du Canada une hausse de sa part des profits de un point de pourcentage engendrerait une augmentation de 0,15 point de pourcentage du PIB comme le montre le tableau 8 (colonne e), mais si tous les pays faisaient de même, cela mènerait à une chute de la croissance canadienne de 0,27 point de pourcentage (comme l'indique la colonne g, en incluant l'effet multiplicateur).

Ainsi il est clair que la relance de l'économie mondiale ne peut passer par la modération salariale, les tenants de cette approche souffrant d'une double erreur de composition: ce qui est vrai pour une entreprise n'est pas nécessairement vrai pour toutes les entreprises; et ce qui est vrai pour un pays pris individuellement n'est pas nécessairement vrai pour l'ensemble de l'économie mondiale. Les résultats du tableau 8 ont des implications considérables. Ils mettent en lumière les effets favorables des augmentations salariales comme composante essentielle de la demande globale, et ils font ressortir la nécessité de la coopération internationale pour empêcher la chute des salaires. Prévenir une telle chute est d'autant plus nécessaire que les économistes sont maintenant conscients que la politique monétaire est complètement impuissante lorsque les taux d'inflation avoisinent le zéro, puisque les banques centrales ne peuvent alors suffisamment abaisser les taux d'intérêt réels, le taux d'intérêt nominal ne pouvant pas baisser plus bas que zéro. Il ne reste que les politiques budgétaires et les politiques de répartition des revenus.

\section{Les régimes de productivité}

Jusqu'à maintenant, nous n'avons considéré que les effets sur la demande globale des changements dans la répartition du revenu. Cependant, à long terme, le niveau de vie d'une population dépend essentiellement de sa productivité, plus précisément la productivité par travailleur ou par heure de travail. Son évolution au cours du temps va donc dépendre du taux de progrès technique et donc du taux de croissance de la productivité. On peut faire pour la productivité les mêmes distinctions que nous avons faites pour la demande. Ainsi, on dira qu'une économie se situe dans un régime de productivité mené par les profits lorsqu'une augmentation de la part des profits conduit à une amélioration de la productivité ou à une accélération 
Tableau 8

Effets de l'augmentation d'un point de pourcentage de la part des profits sur la demande intérieure et sur la demande totale

\begin{tabular}{|l|c|c|c|c|c|c|}
\cline { 2 - 7 } & C & $\mathbf{I}$ & $\mathbf{E X N}$ & $\begin{array}{c}\text { Demande } \\
\text { intérieure }\end{array}$ & $\begin{array}{c}\text { Demande } \\
\text { totale }\end{array}$ & $\begin{array}{c}\text { Effet simultané } \\
\text { sur la demande } \\
\text { totale }\end{array}$ \\
\cline { 2 - 7 } & $\mathbf{a}$ & $\mathbf{b}$ & $\mathbf{c}$ & $\mathbf{d}(\mathbf{a + b})$ & $\mathbf{e}(\mathbf{a + b + c})$ & $\mathbf{g}$ \\
\hline Zone euro-12 & $-0,439$ & 0,299 & 0,057 & $-0,140$ & $-0,084$ & $-0,245$ \\
\hline Allemagne & $-0,501$ & 0,376 & 0,096 & $-0,125$ & $-0,029$ & - \\
\hline France & $-0,305$ & 0,088 & 0,198 & $-0,217$ & $-0,020$ & - \\
\hline Italie & $-0,356$ & 0,130 & 0,126 & $-0,226$ & $-0,100$ & - \\
\hline Royaume-Uni & $-0,303$ & 0,120 & 0,158 & $-0,183$ & $-0,025$ & $-0,214$ \\
\hline États-Unis & $-0,426$ & 0,000 & 0,037 & $-0,426$ & $-0,388$ & $-0,921$ \\
\hline Japon & $-0,353$ & 0,284 & 0,055 & $-0,069$ & $-0,014$ & $-0,179$ \\
\hline Canada & $-0,326$ & 0,182 & 0,266 & $-0,144$ & 0,122 & $-0,269$ \\
\hline Australie & $-0,256$ & 0,174 & 0,272 & $-0,082$ & 0,190 & 0,172 \\
\hline Turquie & $-0,491$ & 0,000 & 0,283 & $-0,491$ & $-0,208$ & $-0,717$ \\
\hline Mexique & $-0,438$ & 0,153 & 0,381 & $-0,285$ & 0,096 & $-0,111$ \\
\hline Corée du Sud & $-0,422$ & 0,000 & 0,359 & $-0,422$ & $-0,063$ & $-0,864$ \\
\hline Argentine & $-0,153$ & 0,015 & 0,192 & $-0,138$ & 0,054 & $-0,103$ \\
\hline Chine & $-0,412$ & 0,000 & 1,986 & $-0,412$ & 1,574 & 1,115 \\
\hline Inde & $-0,291$ & 0,000 & 0,310 & $-0,291$ & 0,018 & $-0,027$ \\
\hline Afrique du Sud & $-0,145$ & 0,129 & 0,506 & $-0,016$ & 0,490 & 0,390 \\
\hline
\end{tabular}

Notes: $\mathrm{C}=$ consommation $; \mathrm{I}=$ investissement $\mathrm{EXN}=$ exportations nettes. La colonne $\mathrm{g}$ tient compte de l'effet multiplicateur.

Source: Özlem Onaran et Giorgos Galanis, «Is aggregate demand wage-led or profit-led? National and global effects», Conditions of Work and Employment Series working paper No 40, Bureau International du Travail, Genève, 2012. La colonne d a été rajoutée par mes soins.

du taux de croissance de la productivité. On dira qu'on est dans le cas d'un régime de productivité mené par les salaires dans le cas inverse comme l'illustre le tableau 9. En général, lorsqu'on ne considère que ces effets directs, la plupart des économistes s'accordent pour dire que c'est le régime de productivité mené par les salaires qui prédomine, car outre l'élimination des entreprises les moins productives, orthodoxes et hétérodoxes s'entendent pour dire que la hausse des salaires réels va inciter les entreprises à adopter des méthodes de production plus mécanisées, dont la productivité par travailleur sera plus élevée. 
La hausse des salaires réels implique cependant aussi des effets indirects sur la productivité. En effet, comme nous l'avons vu dans la section précédente, les changements dans la répartition des revenus vont mener à une accélération ou à une décélération du taux de croissance de la demande globale. Or il existe une relation empirique bien connue, la relation dite de Kaldor-Verdoorn, selon laquelle une croissance plus rapide de l'économie engendre une croissance plus rapide de la productivité. Dans le cas d'un régime de demande mené par les salaires, la hausse des salaires réels aura donc un effet amplifié sur la productivité, grâce aux effets directs et indirects positifs. Par contre, dans le cas d'un régime de demande mené par les profits, l'effet direct d'une hausse des salaires sur la productivité sera diminué par l'effet indirect, puisque la hausse des salaires réels entrainera une baisse du taux de croissance de la demande, ce qui ralentira à son tour le taux de croissance de la productivité, si bien que l'effet global pourrait même être négatif. De façon générale, on peut conclure que la hausse des salaires réels ou de la part des salaires dans le revenu national aura un impact favorable sur le taux de croissance de la productivité, sauf si le régime de demande est fortement mené par les profits, et donc qu'une telle hausse des salaires aura un impact positif sur le niveau de vie et le pouvoir d'achat de la population.

Sachant que la grande majorité des pays participent d'un régime de demande mené par les salaires, et que c'est aussi le cas au niveau mondial ou si l'on ne considère que les effets sur la demande intérieure, on pourrait croire que la hausse des salaires réels pourrait constituer la panacée permettant de relancer l'économie mondiale et de rétablir le plein emploi. Les choses sont cependant plus compliquées. À long terme, pour que l'emploi s'accroisse, il faut que le taux de croissance de la demande globale et de la production soit plus élevé que le taux de croissance de la productivité par travailleur, car la hausse de la productivité permet de produire autant qu'avant mais avec moins de travailleurs. Et à long terme, pour que le taux de chômage diminue (ou pour que le taux d'emploi augmente), il faut que le taux de croissance de la demande surpasse la somme du taux de croissance de la productivité par travailleur et du taux de croissance de la main-d'œuvre disponible. Cependant, rien ne garantit que tel soit le cas, quel que soit le régime économique existant. La hausse des salaires réels, même en supposant qu'elle ait des effets positifs sur la demande, permettra-t-elle de hausser l'emploi et de parvenir au plein emploi? La réponse à cette question est purement empirique, et dépend encore une fois de la valeur des paramètres structurels de l'économie. 
Tableau 9

La structure économique des régimes de productivité menés par les profits et les salaires

\begin{tabular}{|l|l|l|}
\cline { 3 - 3 } \multicolumn{1}{l|}{} & \multicolumn{1}{c|}{ Régime de productivité } \\
\hline \multirow{4}{*}{$\begin{array}{l}\text { Structure } \\
\text { économique par les } \\
\text { profits }\end{array}$} & $\begin{array}{l}\text { Une croissance plus rapide des salaires réels ou } \\
\text { un accroissement de la part des salaires ralentit la } \\
\text { croissance de la productivité }\end{array}$ \\
\cline { 3 - 4 } & \multirow{2}{*}{$\begin{array}{l}\text { Menée par les } \\
\text { salaires }\end{array}$} & $\begin{array}{l}\text { Une croissance plus rapide des salaires réels ou } \\
\text { un accroissement de la part des salaires restreint } \\
\text { l'investissement dans le progrès technique }\end{array}$ \\
\cline { 3 - 4 } & $\begin{array}{l}\text { Une croissance plus rapide des salaires réels ou un } \\
\text { accroissement de la part des salaires accélère la } \\
\text { croissance de la productivité }\end{array}$ \\
\cline { 3 - 4 } & $\begin{array}{l}\text { Une croissance plus rapide des salaires réels ou un } \\
\text { accroissement de la part des salaires accroît l'effort } \\
\text { des travailleurs et accélère l'investissement dans le } \\
\text { progrès technique }\end{array}$ \\
\hline
\end{tabular}

Servaas Storm et Ro Naastepad ${ }^{14}$ ont consacré l'un des modules de notre recherche à tenter de répondre à cette question. Sur la base de ce qu'ils considèrent être les faits stylisés concernant l'effet direct sur la productivité, l'effet sur la demande et l'effet Kaldor-Verdoorn, Storm et Naastepad estiment qu'une augmentation de un point de pourcentage de la part des salaires dans le revenu national permettra d'augmenter le taux de croissance de l'emploi de 0,3 point de pourcentage en omettant les effets liés à la productivité, comme on l'a fait dans la précédente section; mais quand on tient compte de ces effets directs et indirects sur le taux de croissance de la productivité, le taux de croissance de l'emploi diminue d'environ 0,3 point de pourcentage quand la part des salaires augmente d'un point de pourcentage. Ainsi la hausse des salaires, dans la grande majorité des économies avancées, a un impact positif à la fois sur le taux de croissance de l'activité économique et sur le taux de croissance de la productivité, mais il aurait un effet négatif sur le taux de croissance de l'emploi. Il suit que si des politiques de répartition favorables aux travailleurs auront un impact positif sur la demande globale et sur le niveau de vie des travailleurs ayant un emploi, elles auront un impact négatif sur la création d'emplois. Tenir compte de l'impact des hausses de salaire sur la productivité complique donc passablement les conseils que l'on peut offrir à la gent politique!

14. Servaas Storm et C. W. M. Naastepad, «Wages-led or profit-led supply: wages, productivity and investment», Conditions of Work and Employment Series working paper No 36, Bureau International du Travail, Genève, 2012. 


\section{Conclusion}

Malgré les effets négatifs d'une hausse des salaires réels sur l'emploi, Storm et Naastepad continuent à rejeter les politiques fondées sur la modération salariale. Ils constatent que les taux de chômage dans leur propre pays, les Pays-Bas, ont chuté à des niveaux très faibles pendant que les salaires stagnaient et que la part des salaires chutait, mais ils constatent aussi que ce plein emploi s'est accompagné d'une stagnation du niveau de vie, rendant impossible la réduction des heures de travail à pouvoir d'achat constant. On a aussi observé un phénomène similaire au Canada dans les années 2000, avec des taux de chômage relativement bas mais sans hausse marquée de la productivité et du pouvoir d'achat.

La solution à ce dilemme est assez évidente : pour poursuivre une croissance équilibrée, menée par une consommation induite par les salaires allant aux travailleurs ordinaires, ou pour pouvoir diminuer le temps de travail, sans avoir recours à un solde commercial en surplus, il est nécessaire que l'État fasse explicitement la promotion du plein emploi et qu'il poursuive des politiques budgétaires expansionnistes, accompagnées par des politiques fiscales progressistes, qui taxent davantage les contribuables à revenus élevés dont les propensions à consommer sont les plus faibles. De plus, comme le rapportent Storm et Naastepad, des études récentes ont montré que rendre plus flexible le marché du travail, en affaiblissant les protections des travailleurs, ne contribuait aucunement à augmenter les taux de croissance de la productivité et de la demande, si bien que les gouvernements feraient mieux de renforcer les institutions et les lois favorables aux travailleurs et aux organisations syndicales. Enfin, et même si ceci semble inconcevable pour le moment, il est clair que la bonne gouvernance des entreprises, financières et non financières, devra un jour passer par l'imposition de normes maximales de dispersion des rémunérations et par l'élimination des bonus à la prétendue performance ${ }^{15}$.

Selon Hein, les gouvernements doivent s'affairer à mettre en ouvre un New Deal keynésien, qui s'attaque aux trois causes majeures de la crise actuelle: "l'inefficacité de la régulation des marchés financiers, l'augmentation de l'inégalité de la distribution des revenus et la montée des déséquilibres au niveau mondial (et dans la zone euro) ${ }^{16}{ }^{»}$. Pour ce faire, outre les modifications aux politiques budgétaires et fiscales déjà mentionnées, il faudra réguler à nouveau le secteur financier, notamment le secteur dit

15. C'est ce que proposent aussi Yvan Allaire et Mihaela Firsirotu, Playdoyer pour un nouveau capitalisme: sur les causes profondes des crises financières et sur les moyens de s'en sortir, Montréal, Institut sur la gouvernance d'organisation privée et publique, 2010, ch. 8.

16. Eckhard Hein, «Redistribution, déséquilibres mondiaux et crise économique et financière: playdoyer pour un New Deal keynésien», Journal international de recherche syndicale, vol. 3, nº 1, 2011, p. 73. 
du shadow banking, et en diminuer l'importance afin de prévenir des crises financières futures et afin de diminuer les inégalités de revenus. Il faudra aussi que les principaux acteurs économiques, les gouvernements mais aussi les syndicats, prennent conscience des erreurs de composition associées aux politiques de modération salariale qui ont pour objectif de ravir les marchés extérieurs aux pays qui poursuivent des politiques expansionnistes, afin que les syndicats mettent davantage de pression pour obtenir des hausses salariales substantielles dans les pays qui ont la chance de bénéficier d'un solde du compte courant en surplus.

Deux visions s'affrontent. D'un côté, il y a le point de vue de certaines grandes institutions comme l'OCDE et la Banque centrale européenne (BCE), selon lesquels moins de réglementation sur tous les marchés, y compris le marché du travail, permettrait à l'économie de retrouver son plein potentiel, grâce à la plus grande flexibilité du marché du travail et à la baisse des salaires qui y serait associée. De l'autre côté, il y a le point de vue présenté ici et défendu par d'autres grandes institutions, comme la CNUCED et l'Organisation Internationale du Travail (OIT), selon lesquelles la baisse des salaires aurait des effets néfastes sur l'ensemble de l'économie mondiale. Comme le dit un chercheur de l'OIT,

«Si tous les pays réduisent simultanément les salaires pour rester compétitifs, l'avantage concurrentiel disparaît et le poids des diminutions de salaires pratiquées à travers le monde sur la consommation risque d'induire une dépression de la demande globale et de l'emploi à l'échelle mondiale ${ }^{17}{ }$.

La sortie de la crise mondiale provoquée par l'instabilité financière passe par une augmentation de la part des salaires, et non par la modération salariale, aussi étrange que cela puisse paraître à ceux qui ignorent les paradoxes macroéconomiques engendrés par les modifications associées à la demande globale.

17. Voir le communiqué de l'OIT intitulé «Diminuer les salaires peut entraver la croissance», 10 août 2012, www. ilo.org/global/about-the-ilo/newsroom/news/WCMS_187221/lang--fr/index.htm. Voir aussi le rapport de l'OIT, Rapport mondial sur les salaires 2012/13: salaires et croissance équitable, Genève, Organisation internationale du travail, 2013, www.lo.org/wcmsp5/groups/public/---dgreports/---dcomm/---publ/documents/publication/ wcms_213108.pdf 